\title{
Mind, Body, and Soul
}

Paul T E Cusack

BScE, DULE, 23 Park Ave., Saint John, NB E2J 1R2, Canada

Corresponding Authors: Paul T E Cusack, BScE, DULE, 23 Park Ave., Saint John, NB E2J 1R2, Canada.

Received Date: September 01, 2020; Accepted Date: September 07, 2020; Published Date: September 10, 2020. Citation: Paul T E Cusack, (2020) The Mind, Mental Constructs \& the Cosmic Pyramid. J Clinical Case Reports and Studies, 1(4): Doi: 10.31579/2690-8808/028

Copyright: ( $)$ 2020. Paul T E Cusack. This is an open-access article distributed under the terms of the Creative Commons Attribution License, which permits unrestricted use, distribution, and reproduction in any medium, provided the original author and source are credited.

\section{Abstract}

In this paper we uses AT Math and electrical engineering equations to prove that the mind, body and soul evolved to match up with the universal signal. Questions as to whether there is a creator or not is mentioned as well as the nature of consciousness.

Keywords: Mind; Body; Soul; Consciousness; AT Math

\section{Introduction}

The Bible tells us "In Him we live and move and have our being." But to believe that the universe is synonymous with God is pantheism. Of course, energy cannot be created nor destroyed. Thus, it is eternal. God is eternal. I $\mathrm{f}$ God is infinite, does this mean that He is everywhere omnipresent? These are questions I cannot answer. But we can answer basic questions about the universe including the human mind

The mind, body and soul evolved to sense the universal signal of 1/sqrt3. .We use basic electrical engineering equations to show that there is a soul. We provide equations for the mind, body, and soul.

“.... according to Rosenthal, when a mental state is accompanied by a conscious or unconscious higher-order thought to the effect that one has it, this ipso facto guarantees that the mental state is a conscious state." [1] If Second -Order thoughts imply conscious states, and a thought is simply energy, then could the universe be alive? In terms of mathematics, second order thoughts are the same as the Second Order Differential equation called the Clairnaut Equation. It governs the universe.

If the universe evolved on its own from the zero vector, then humans are simply machines made up of biological circuits that self-reflect thus know they are alive. If according to Rosenthal, a second order thinker must be conscious, then machines with AI are alive. But those machines have creators i.e., engineers Therefore, since humans are second order thinkers, we must also have a creator viz God.

$\mathrm{d}^{2} \mathrm{E} / \mathrm{dt}^{2}-\mathrm{G}=0$

$\mathrm{y}=\mathrm{y}^{\prime}=2$

$\mathrm{SE}=\mathrm{t}^{2}-\mathrm{t}-1$

$\mathrm{SE}=2 \mathrm{t}-1$

$\mathrm{SE}^{\prime}=2$

$\mathrm{SE}=2(2)-1=3=\mathrm{c}$

$\mathrm{SE}^{\prime}=(3)^{2}-3-1=5=\mathrm{E}$ when $\mathrm{SE}=\mathrm{SE}{ }^{\prime} \Rightarrow \mathrm{y}=\mathrm{y}^{\prime}$ $\iint \mathrm{d}^{2} \mathrm{E} / \mathrm{dt}^{2}=\mathrm{E}=\iint \mathrm{G}=\mathrm{G}^{3} / 3=2^{3} / 3=8 / 3=$ S.F.

Is the universe alive?

The Universe is made up of ultimately Energy. So are thoughts. Consider the Neuron Firing Energy. [2] However, just because the universe has second order energy does not men it is alive. Neither is a machine that has second order thought. Human beings have second and third order thoughts. And we know it. However, we are alive and thus we are different. That difference is the soul.

$\mathrm{E}=(1-\operatorname{Ln} 0.11027)^{7}$

$=105.6$

$=\mathrm{V}=|-70 \mathrm{mV}|+35 \mathrm{mV}$ Signal of the nervous system.

$\mathrm{E}=1 / \mathrm{t} \mathrm{E}=1=1 / 1=1$

Mind:

$\mathrm{E}=(1-\operatorname{Lnt})$

$1=(1-\operatorname{Ln} 1)$

$\mathrm{t}=1$

Body:

$\Sigma$ Senses $=1$

Soul:

$\mathrm{SE}=\mathrm{t}^{2}-\mathrm{t}-1=1$

$\mathrm{t}=2 ;-1$

Now the universe is described by Euler's Identity:

$\sin ^{2} \theta+\cos ^{2} \theta=1$

$\mathrm{y}=\mathrm{y}$

$\sin \theta=\cos \theta$ 
$\theta=\pi / 4=\mathrm{t} / 4$

Now the Universe can be described by the Universal Parametric Equation [4].

$(x, y)=(t, E)=\sin t+1 / 3 \cos [17 t+\pi / 3] ; \sin [17 t+\pi / 3]$

Note the following values:

- $\quad 17=8$ th Prime Number

- $1 / 3=1 / \mathrm{c}$

- $\pi / 3=60^{\circ}=1 / 6 \times 360^{\circ}$

Gauss' Equation of Primes:

$\operatorname{Lim} x \rightarrow \infty \pi(x) /[x / \operatorname{Ln} x]=1$

$8 /[17 / \operatorname{Ln} 17]=8 / 6=4 / 3=\mathrm{s}=\mathrm{i}$

$\mathrm{s}=|\mathrm{E}||\mathrm{t}| \sin \theta$

$=(1)(1) \sin 60^{\circ}$

$=0.866$

Now

$8 / 6$

$=1 / 6 \times 8$

$=1 / 6 \times 1 / \mathrm{E}$

$=1 / 6 \times \mathrm{x}$

$=60^{\circ} / 360^{\circ} \mathrm{t}$

$=\pi / 3 \times 1 / \pi$

$=1 / 3$

$=1 / \mathrm{c}$

$\operatorname{Lim} x \rightarrow \infty 1 / c=1$

$\int \operatorname{Lim} x \rightarrow \infty 1 / c=\int 1 d x$

Ln $c=x+\mathbb{C}$

Ln $3=x+\mathbb{C}$

$1.0986=\mathrm{x}+\mathbb{C}$

But $\mathrm{x}=\mathrm{t}=1$

$1.0986=1+\mathbb{C}$

$\mathbb{C}=0.0986$

$\mathrm{E}=(1-\operatorname{Ln} \mathrm{t})^{7}$

$=(1-0.986)^{7}$

$=901.4$

$=3^{2}$

$=\mathrm{c}^{2}$

$\mathrm{M}=1$

$\mathrm{E}=\mathrm{c}^{2}=\mathrm{Mc}^{2}$

$\mathrm{M}=1$

$\mathrm{t}=\mathrm{KE}=1 / 2 \mathrm{Mv}^{2}$

$=1 / 2(1(1 / 2)$
$=\mathrm{M} / 4$

$\mathrm{t}=0.250 \mathrm{v}=\mathrm{d} / \mathrm{t}=0.011027=\mathrm{d} / 250$

Hearing: "Faith comes from hearing."

$\mathrm{d}=275.7$

$150.7+125=255.7$ (Double Line path in Figure 1).
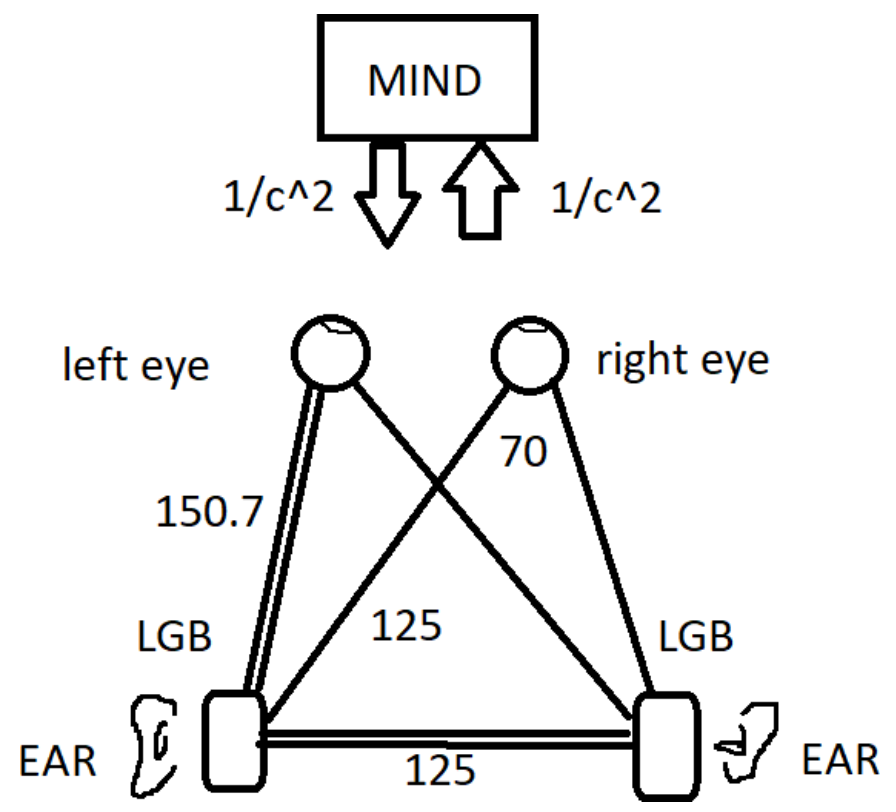

Figure 1. Path of consciousness 
$=17 / 72$

$=17 /\left[\mathrm{t} \times 1 / \mathrm{c}^{2}\right]$

$17\left(3^{2}\right)(0.011027)$ (Neuron Fire)

$=1664$

$=1 / 601=1 / 6=60 / 360=\pi / 3$

$\mathrm{SE}=1 / 6=1 /$ Evil=Good!

$8 / 6=1 / 6 \times 8 / 1$

$=1 / 6 \times \mathrm{t}$

$=1 / 6 \times 1 / \mathrm{E}$

$=1 /(6 \mathrm{E})$

$=4 / 3$

$1 / \mathrm{E}=2$

$\mathrm{E}=1 / 2$

$\mathrm{E}=1 / \mathrm{t}$

$\mathrm{t}=2=\mathrm{L}=\mathrm{y}=\mathrm{y}$,

$E=(1-\operatorname{Ln} t)=1 / 2$

Ln $\mathrm{t}=1 / 2$

$t=6.06$

$\mathrm{E}=165$

$165=-150.7=0.143=1 / 7$

$165 \times 7=1155==1 / 0.866=1 / \sin 60^{\circ}$

Now,

$8 / 6 \times 1 / 6=222=1 / 9(2)=\mathrm{ML}$

$\mathrm{ML}=0.222$

ML-SE $=0.222-1 / 6=0.5555=1 / 180^{\circ}=1 / \pi=$ freq of the human mind.

$\mathrm{TE}=\mathrm{PE}+\mathrm{KE}+\mathrm{SE}$

$=\mathrm{Mc}^{2}+\mathrm{t}+\mathrm{t}^{2}-\mathrm{t}-1$

$1=\mathrm{Mc}^{2}+\mathrm{t}^{2}-1$

$2=\mathrm{Mc}^{2}+\mathrm{t}^{2}$

$\mathrm{M}=1$

$2=\mathrm{c}^{2}+\mathrm{t}^{2}$

Pythagoras

$\mathrm{c}=\mathrm{d} / \mathrm{t}=2 / \mathrm{t}$

$\mathrm{c}=\mathrm{v}=\mathrm{d} / \mathrm{t}$

$(\mathrm{d} / \mathrm{t})^{2}+\mathrm{t}^{2}=(\sqrt{ } 2)^{2}$

$\mathrm{dt}+1 /\left[\mathrm{t}^{2} \mathrm{t}=(2\right.$

$\mathrm{dt}=1=\mathrm{t}^{2}(\sqrt{ } 2)^{2}$

$\mathrm{dt}+1=\mathrm{t}^{2}(\sqrt{2} \sqrt{ })^{2}$

$(\sqrt{2})^{2} \mathrm{t}^{2}-\mathrm{dt}-1=0$

$t^{2}-d(\sqrt{2})^{2}-1=0$

$\mathrm{t}^{2}-\mathrm{t}-1=\mathrm{SE}$

Roots $\mathrm{t}=1.618 ;-0.618$

Golden Mean parabola

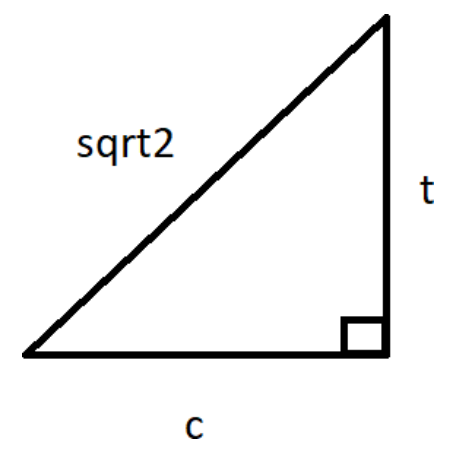

Figure 2

$c^{2}+t^{2}=(\sqrt{ } 2)^{2}$

$(2 / t)^{2}+t^{2}=2$

$4 / t^{2}+t^{2}=2$

$\left(4+t^{2}\right) / t^{2}=2$

$4=2 \mathrm{t}-\mathrm{t}$

$4=\mathrm{t}$

$\mathrm{t}=2$

$\mathrm{c}^{2}+\mathrm{t}^{2}=(\sqrt{ } 2)^{2}$

$c^{2}=2-4=-2$

$\mathrm{c}=\sqrt{ }(-2)$

$\mathrm{c}=(-0.618)(1.412)$

$=0.873$

$1 / \mathrm{c}=1 / 0.874=1 / 1.1441$

$\mathrm{s}=|\mathrm{e}||\mathrm{t}| \sin 60^{\circ}$

$(0.865)(2)(0.866$

$s=149.8=1 / 6.67=1 / \mathrm{G}$

$\mathrm{d}^{2} \mathrm{E} / \mathrm{dt}-\mathrm{G}=0$

$\mathrm{dE} / \mathrm{dt}-1 / \mathrm{s}=0$

$\iint \mathrm{d}^{2} \mathrm{E} / \mathrm{dt}^{2}=\iint 1 / \mathrm{s}=\int \operatorname{Ln} \mathrm{x}$

$\mathrm{E}=\mathrm{x} \operatorname{Ln} \mathrm{x}-\mathrm{x}+\mathbb{C}$

$E=(1-\operatorname{Ln} t)$

$=1-\operatorname{Ln} 1.114$

$=865$

$\sim \sin 60^{\circ}$ 
$\mathrm{E}=\sin 60^{\circ}=\mathrm{xLn} \mathrm{x}-\mathrm{x}+\mathbb{C}$

$=2 \operatorname{Ln} 2-2$

$0.866=-0.614+\mathbb{C}$

$\mathrm{t}=147.9 \sim \mathrm{s}$

$\sin 60^{\circ}=x \operatorname{Ln} x-x+\mathbb{C}$

$0.866=x \operatorname{Ln} x-x+\mathbb{C}$

$0.866=\mathrm{x} M-\mathrm{x}$

$M=4$

$0.866=4 \mathrm{M}-\mathrm{x}+\mathbb{C}$

$0.866=3 x+\mathbb{C}$

$\mathrm{x}=2.886+\mathbb{C}$

2.886Ln $2.886-2.886+\mathbb{C}$

$=1.727+\mathbb{C}$

$\sqrt{3}=\mathbb{C}$

$\mathbb{C}=0.866=\sin 60^{\circ} \mathbb{C}=\sin 60^{\circ}-\sqrt{ } 3$

$=$ E-Eigenvector

$=\mathrm{E}-\mathrm{t}$

C

$\sin 60^{\circ}=x \operatorname{Ln} x-x+\mathbb{C}$

$=\mathrm{t} L n \mathrm{t}-\mathrm{t}+\mathrm{E}-\mathrm{t}$

$0.866=t(M)-2 t+E$

$\mathrm{tM}=2 \mathrm{t}$

$M=2$

$\mathrm{M}=\mathrm{t}=\mathrm{Ln} \mathrm{t}$

$\mathrm{t}^{2}-\mathrm{t}-\mathbb{C}=\sin 60^{\circ}$

$\mathrm{t}^{2}-\mathrm{t}-2(0.866)-\sqrt{3}$

$\mathrm{t}^{2}-\mathrm{t}-134-\sin 60^{\circ}=0$

$\mathbb{C}=\left(1-\sin 60^{\circ}\right)$

$=0.134=1 / 746$

$\mathrm{E}=2 \mathrm{Ln} 2-2+0.134$

2Ln 2-0.132

$=663$

$\mathrm{t}=150.6$ Cf. 150.7 Distance to ear.

$\mathrm{t}=1 / \mathrm{G}$

$\mathrm{E}=\mathrm{G}$

And,

$t^{2}-t-1=E$

$1.508^{2}-1.508-0.1=0.666=\mathrm{G}$

$\mathrm{E}=\mathrm{G}$

$\mathrm{d}^{2} \mathrm{E} / \mathrm{dt}^{2}-\mathrm{G}=0$
$\mathrm{E}=\mathrm{G}^{3} / 3$

$E=E^{3 / 3}$

$E^{2}=3$

$E=\sqrt{ } 3=1 / t=1 / \sqrt{ } 3=$ Universal Signal

$\mathrm{t}^{2}-\mathrm{t}-1=\mathrm{E}$

$(1 / \sqrt{ } 3)^{2}-(1 / \sqrt{ } 3)-1=E$

$\mathrm{E}=\mathrm{SE}=-1.244=-1 / 804$

$\mathrm{SE}=2 \mathrm{t}-1$

$=2(1 / \sqrt{ } 3)-1$

$=1.1547-1$

$=\left(1 / \sin 60^{\circ}\right)-1$

$=\csc 60^{\circ}-1$

$\mathrm{SE}=2$

$\mathrm{TE}=\mathrm{PE}+\mathrm{KE}+\mathrm{SE}$

$=\mathrm{Mc}^{2}+(1 / \sqrt{ } 3)^{2}-1$

$=\mathrm{Mc}^{2}-0.666$

$\mathrm{TE}=\mathrm{PE}-\mathrm{G}$

$\mathrm{TE}=\mathrm{Mc}^{2}-\mathrm{F} / \mathrm{G}$ Newton's Gravitational Formula $\mathrm{F}=\mathrm{GM}$

$100 \%=1=\left[\mathrm{M}^{2} \mathrm{c}^{2}-\mathrm{F}\right] / \mathrm{M}$

$\mathrm{M}=\mathrm{M}^{2} \mathrm{c}^{2}-\mathrm{F}$

$\mathrm{M}=\mathrm{M}^{2} \mathrm{G}^{2}-\mathrm{GM}-\mathrm{M}=0$

$\mathrm{M}=1$

$1^{2} \mathrm{c}^{2}-\mathrm{G}(1)-1=0$

$\mathrm{c}^{2}-\mathrm{G}-1=0$

$\mathrm{c}^{2}=1+\mathrm{G}$

$c^{2}=1.666=1 / 6$

$c=1 / \sqrt{ } 6 \sqrt{ }=0.408$

$c^{2}-\mathrm{G}=1$

$\mathrm{c}^{2}-\mathrm{G}-1=0$

$c^{2}-E=100 \%$

$\mathrm{PE} / \mathrm{M}-\mathrm{E}=1$

$\mathrm{M}=1$

$\mathrm{PE}-\mathrm{E}=1$

$-E=1-P E$

$\mathrm{E}=\mathrm{PE}-1$

$\sqrt{ } 3=$ PE-1

$P E=\sqrt{ } 3+1$ 


\section{$2.73=\mathrm{PE}=\mathrm{Mc}^{2}$}

$\mathrm{M}=3.04$

$\sim \mathrm{c}$

$\mathrm{PE}=\mathrm{Mc}^{2}$

$=\mathrm{c} \cdot \mathrm{c}^{2}$

$=\mathrm{c}^{3}$

$=2732$

$\mathrm{PE}=1+\sqrt{3}$

$\mathrm{TE}=\mathrm{PE}+\mathrm{KE}+\mathrm{SE}$

$1=(1+\sqrt{ } 3)+\mathrm{KE}+(-1.244)$

$-\sqrt{ } 3+1.244=\mathrm{KE}=1 / 2 \mathrm{Mv}^{2}$

$\mathrm{KE}=-195.2$

$\mathrm{E}=1 / \mathrm{t}$

$=1 /-195.2=512=\mathrm{t}$

$\mathrm{t}=512 \sim 51$

Hearing:

$\mathrm{d}_{\mathrm{s}}=12.5 \mathrm{~cm} \sim 5$ inches Distance from eardrum to LGB.

$\mathrm{v}=\mathrm{d} / \mathrm{t}$

$0.011027=12.5 / \mathrm{t}$

$\mathrm{t}=113.4$

$\mathrm{V}=\mathrm{iR}$

$105=\mathrm{i}(0.85)$

$\mathrm{i}=1.243 \sim 1.25$

$\mathrm{P}=\mathrm{Va}$

$=105(125)$

$=257$

$\mathrm{P}=\mathrm{i}^{2} \mathrm{R}$

$=\left(1.243^{2}(0.856)\right.$

$=1.322$

$\mathrm{E}=(1-\operatorname{Ln} \mathrm{t})$

$=(1-\operatorname{Ln} 0.011027)$

$=458$

$\mathrm{t}=218.4-1.243=217.2=1 / 0.460$

$\mathrm{t}^{2}-\mathrm{t}^{2}-1=\mathrm{E}$

$0.460^{2}-0.460-1=1.248 \sim 1.25=\mathrm{E}_{\min }$

$\mathrm{vs}=343 \mathrm{~m} / \mathrm{sec}$

$\mathrm{v}=\mathrm{d} / \mathrm{t}$
$343=(114.3)+60.6] /$ ts

ts $=50.99 \sim 51$

$\mathrm{t}-\mathrm{KE}=\mathrm{SE}$

$(0.51)^{2}-(0.51)-1=\mathrm{SE}$

$\mathrm{SE}=-1.25=\mathrm{E}_{\min }$

$\mathrm{t}_{\min }=1 / 2$

$\left.\mathrm{SE}^{\prime}=2 \mathrm{t}-1=21 / 2\right)-1=0$

$\mathrm{SE}=2$

$P E=1-\sqrt{ } 3$

$\mathrm{E}=\mathrm{PE}-1$

$1-\sqrt{3}-1$

$=2-\sqrt{3}$

$=2.67=\Pi$ Senses $=\mathrm{SF}$

Mind meets the Body meets the Soul

$\mathrm{S}=\mathrm{E}-\mathrm{M}=1 / 8-1 / 9=1 / 72=\Pi$ Senses $/ \Sigma$ Senses $=2.67$

$-1 / 8=$ Emin

$\left(\mathrm{t}^{2}-\mathrm{t}-1\right)^{-1}-1 / 9=2.67$

$1 / \mathrm{E}^{2}-1 / \mathrm{E}-1 / \mathrm{E}=278$

$\mathrm{t}^{2}-\mathrm{t}-1=359.6$

$\mathrm{t}^{2}-\mathrm{t}-1.359=0$

$\mathrm{t}=3219 ; 222$

$\mathrm{E}=31 ; 45.05$

$\mathrm{E}=(1-\operatorname{Ln} \mathrm{t})^{7}$

$=(1-\operatorname{Ln} 31)^{7}$

$=343^{7}=(\text { speed of Sound })^{7}$

$=5.59$

$\mathrm{t}=178.7$

$\mathrm{t}^{2}-\mathrm{t}-1=3.175 \sim 1 / \pi$

$\mathrm{E}=\left(1-\operatorname{Ln}(0.222)^{7}\right.$

$=1.50^{7}$

$=1.849$

$=\mathbf{1} / \mathrm{G}$

$\mathrm{t}=541$

$\mathrm{t}^{2}-\mathrm{t}-1=-1.248=\mathbf{1 . 2 5}=\mathbf{E}_{\min }$ 


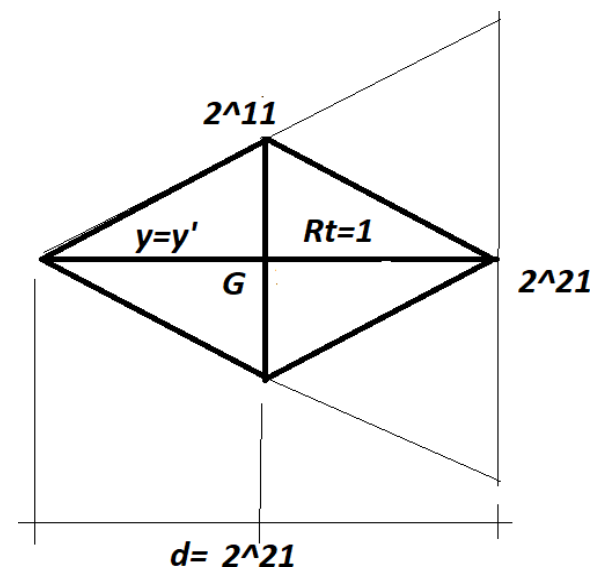

Figure 3. The Mind Binominal Tree.

Vision is inverted on the retina.

$1 / \mathrm{G}=1 / \pi \cdot \chi$

$=\pi / \mathrm{G}=512$

$\mathrm{t}^{2}-\mathrm{t}-1=\mathrm{SE}$

512 $2-512-1$

$=-1.2498$

$\sim-1.25$

$=\mathrm{E}_{\min }$

Nerve Signal

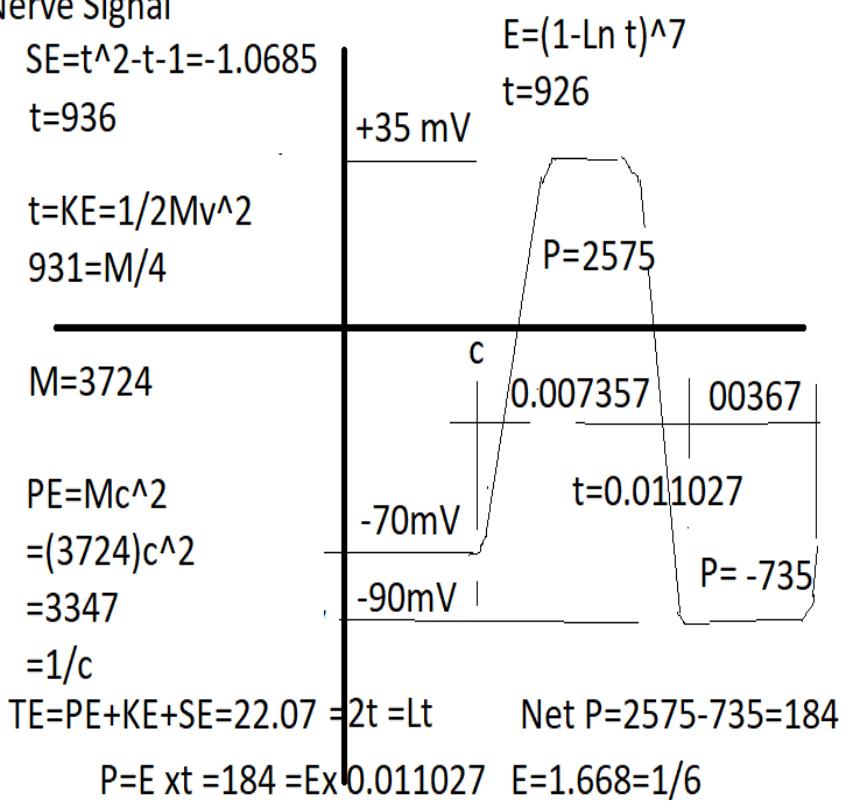

Figure 4. The Nerve Signal
$P=j \wedge 2 R$

22.07=i^2 (3)

$i=2.712^{\sim}$ base $e^{\wedge} 1$

$V=i R$

$e^{\wedge} 0.693=2=L=y=t$

$105=35(R)$

$\mathrm{R}=3$ ohms

$1 / 0.693(-1.25)=1.8037$

$\sim 180=$ Pi rads=t $\max$

$\mathrm{P}=\mathrm{V} \times \mathrm{A}$

$22.07=105 \times A$

$A=21.01 \mathrm{Amps}$

$E=(1-\operatorname{Ln} 180)^{\wedge} 7$

$=539$

539^2-539-1=1.25=Emin

1.602 Coul $x t / 22=0.727 \mathrm{sec}$

Figure 5. Electrical Engineering calculations I

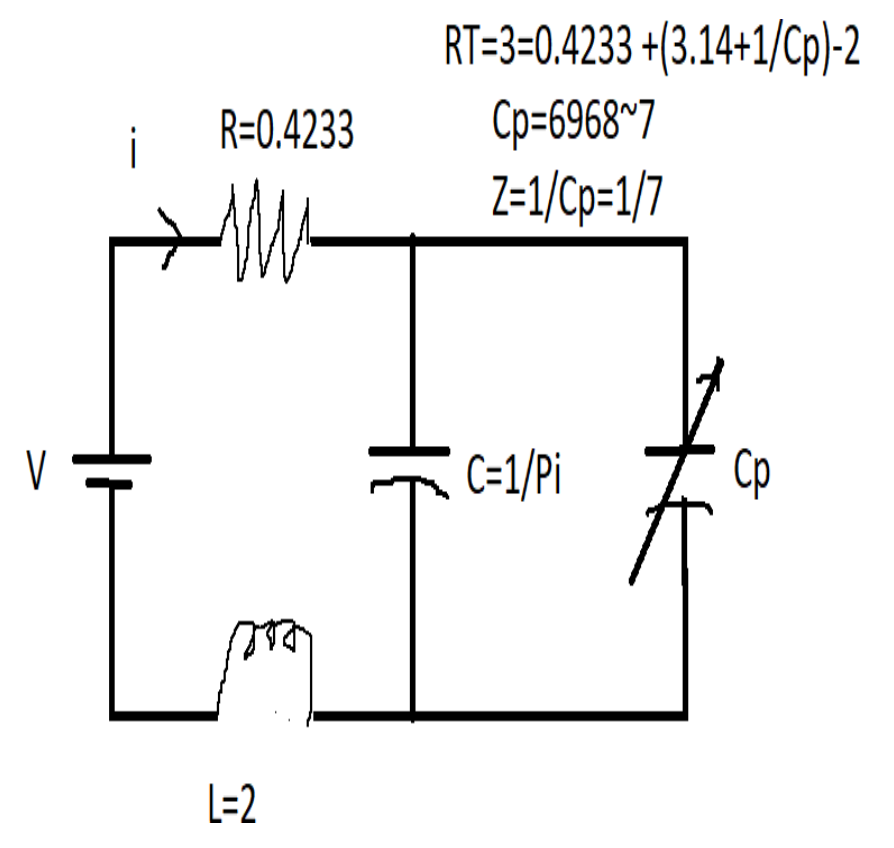

Figure 6. Electric Circuit for the Mind and the Universe 


\section{Amps $x / 1.602$ Coul $=$ \\ 13.109e-}

$13.109 \mathrm{e}-\mathrm{x} 5.11 \mathrm{gm}=67 \mathrm{gm}$

$=0.602$

\section{$P E=\operatorname{Ln} t c^{\wedge} 2$ \\ $=\operatorname{Ln} 397 \times c^{\wedge} 2$ \\ $=8.303$}

$P E=67 \times c^{\wedge} 2$

$1-0.602=397^{\sim} 4$

$$
\begin{aligned}
& E=(1-\operatorname{Ln} t)^{\wedge} 7 \\
& =(1-\operatorname{Ln} 397)^{\wedge} 7 \\
& =157.4 \\
& =P i / 2=90 \mathrm{deg} .
\end{aligned}
$$

to=2/Pi=6.366

Moment of Creation

$$
\begin{aligned}
& S E=t^{\wedge} 2-t-1 \\
& =(6.366)^{\wedge} 2-6.366-1 \\
& =332=1 / 301.5=1 / c \\
& t=K E=1 / 2 M^{\wedge} 2 \\
& M / 4=6.366 \quad M=253
\end{aligned}
$$

Figure 7: Some electrical Calculations II

$\mathrm{TE}=\mathrm{PE}+\mathrm{KE}+\mathrm{SE}$

$=8.303+6.366+0.332$

$=` 15.001$

$150=1 / \mathrm{G}$

John Locke and Robert Boyle concluded that there are primary qualities and secondary qualities called qualia. The primary qualities include: Size, shape, solidity and number and motion. The secondary qualities include sight, sound, taste, hearing and touch. i.e. the senses.

The primary qualities are simply Potential Energy and Kinetic Energy. The secondary qualities are the 5 human senses. So, we can put numbers to them both:

$\mathrm{PE}=\mathrm{Mc}^{2}+\mathrm{Mgh}$

$\mathrm{KE}=1 / 2 \mathrm{Mv}^{2}$

$\Sigma$ Senses $=1 \quad \mathrm{~V}=\mathrm{iR}=4 / 3(\mathrm{R})=4 / 3(1)=4 / 3$

So

Total energy $=\mathrm{TE}=\mathrm{PE}+\mathrm{KE}+\mathrm{V}$

$=\mathrm{M}=1$

$(1) \mathrm{c}^{2}+(1)(6.67)(1)+1 / 2(1)(1 / \sqrt{ } 2)^{2}+4 / 3$

$=9+6.67+1 / 4+4 / 3$

$=1.733$

$=\sqrt{3} \quad$ The Inverse of the Universal Signal

$\mathrm{t}^{2}-\mathrm{t}-1=\mathrm{SE}$

$(\sqrt{3})^{2}-(\sqrt{ } 3)-1=2.67=\Pi$ Senses $/ \Sigma$ Senses

$\mathrm{SE}=\Pi$ Senses $/ \Sigma$ Senses $=\mathrm{SF}$

Now,
$\mathrm{TE}=\mathrm{PE}+\mathrm{KE}+\mathrm{SE}$

$=8.3-3+6.366+0.332$

$=15.001$

$\sim 15.0=1 / \mathrm{G}$

$\mathrm{TE}=\mathrm{TE}+\mathrm{TE}=15.001+1.733$

$=16.7331$

$=1 / 6$

Now,

$\mathrm{V}=\mathrm{iR}$

(1) $\mathrm{V}=4 / 3 \mathrm{SE}$

$\mathrm{TE}=\mathrm{V}(4 / 3(15)=2.000=\mathrm{TE}=\mathrm{y}=\mathrm{y}$,

$\mathrm{SE} / \mathrm{E}=\mathrm{SE} / 5=\mathrm{R}=15 / 5$

$\mathrm{V}=\mathrm{iR}=105=35(\mathrm{R}) \mathrm{R}=3$

$\mathrm{SE} / 5=\mathrm{SE}^{\prime} / 5$

$\{2 \mathrm{t}-1] / 5=15$

$\mathrm{t}=8$

$\mathrm{E}=1 / \mathrm{t}=1 / 8=1.25$

$\mathrm{V} / \mathrm{SE}=4 / 3 / 1=4 / 3$

(1) $\mathrm{V}=4 / 3 \mathrm{SE}$

$(1)(1)=4 / 3(15)=20.000=\mathrm{L}=\mathrm{t}=\mathrm{y}=\mathrm{y}$ '

$\Sigma \mathrm{TE}=150.1+1.733$

$=151.73$

$\Delta$ Poer $/ 2=151.725$

$\mathrm{P}+=(35)(10103) / 2$

$=158.7$

$\mathrm{P}-=(-90)(1103 / 2)$

$=462$

$\Delta \mathrm{P}=158.7-462$

$=3.03$

$\Delta \mathrm{P} / 2=151.725=\sqrt{ } 3+150.1$

$\mathrm{P}=\mathrm{Ext}$

$=1157.73=\mathrm{E} \times 1103$

$\mathrm{E}=137.6=1 / 726 \sim(1 / 8) \times(1 / 9)=$ Mind

$\mathrm{TE}_{1}=\mathrm{PE}+\mathrm{KE}+\mathrm{SE}$

$\mathrm{TE}_{2}=\mathrm{PE}+\mathrm{KE}+\mathrm{V}$

$\mathrm{TE}^{1 / T E^{2}}=[\mathrm{PE}+\mathrm{KE}+\mathrm{SE}] /[\mathrm{PE}+\mathrm{KE}+\mathrm{V}]$

$=\mathrm{SE} / \mathrm{V}$

$=15.001 / 1.733=0.8656=\sim \sin 60^{\circ}$

$\mathrm{s}=|\mathrm{E}||\mathrm{t}| \sin 60^{\circ}$

$s /(E)(t)=\sin 60^{\circ}$ 
$\mathrm{s}=(15.001)(11.027)(0.866)$

$=1 / 698 \sim 1 / 7$

$\mathrm{SE} / \mathrm{V}=\mathrm{SE} / \mathrm{E}=\sin 60^{\circ}$

$\mathrm{SE}=\mathrm{s}=|\mathrm{E}||\mathrm{t}| \sin 60^{\circ}$

$\mathrm{SE}=$ space $=\mathrm{s}$

$\mathrm{SE}=\mathrm{t}^{2}-\mathrm{t}-1=\mathrm{s}=4 / 3$

$\mathrm{t}=21.07 ; 1107$

$2^{21}=2^{\mathrm{t}}=$ Mind

$\mathrm{t}=1107 \sim 1103=$ Nerve Firing.

This is proof that there is a soul (i.e. the gremlins)

This is "a combination of electrochemical happenings in the brain." [1] pg 410

$\Pi$ Senses $=2.67$

"The idea of the self as the centre of negative gravity"

$\mathrm{d}^{2} \mathrm{E} / \mathrm{dt}^{2}-\mathrm{G}=0$

$-d^{2} E / d t^{2}=-G=(1 / t)$

$\mathrm{t}=150.0$ (the self) $=\mathrm{SE} / \mathrm{V}$

$\mathrm{SE}=\mathrm{Vt}=(105.7)(15.01)=1586=$ Moment $=\mathrm{F}$ xd

$=8 / 3)(595)$

$=2.67 \times 601=$

$=\Pi$ Senses $\times 601=1.602=1$ Coulomb $=$ Electron charge

The Inductor:

L/Coulombs $=2 / 1.6027=1.248 \sim 1.25=\mathrm{E}_{\min }$
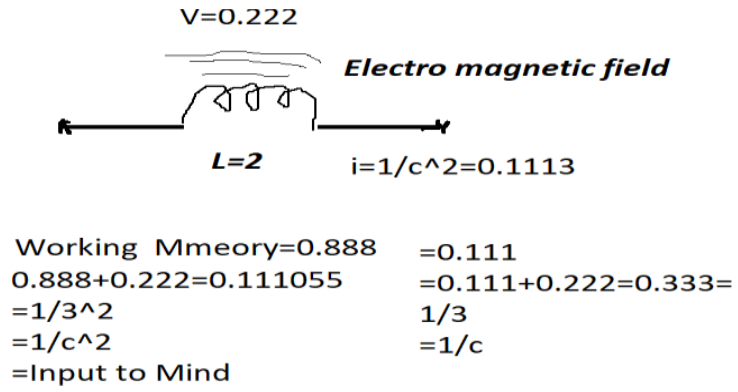

Figure 7: Inductor

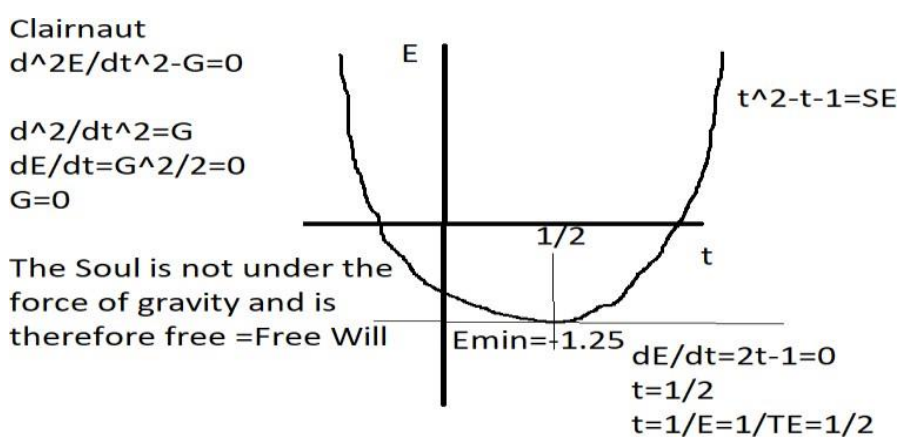

Figure 8: Free Will

$\mathrm{E}=(1-\operatorname{Ln} \mathrm{t})^{7}$

$-1.25^{-1 / 0.93}=1-$ Ln t

$-1.122=-\mathrm{Ln} \mathrm{t}$

$\mathrm{t}=31.03 \sim 31=12$ th Prime number

Gauss' Equation:

$\operatorname{Lim} x \rightarrow \infty \pi(x) / x / \operatorname{Ln} x=1$

$12 /[31 / \operatorname{Ln} 31]=1329$

$1 / 1329=752=\mathrm{t}$

$=1 / 1.77=1 /$ Power.

$\mathrm{s}=|\mathrm{E}||\mathrm{t}| \sin \theta$

$\mathrm{s}^{\prime}=\mathrm{dE} / \mathrm{dt}(\mathrm{dt} / \mathrm{dt} \cos \theta$

$=(1)(1)(1 / 2)$

$=1 / 2=\mathrm{t}_{\min }$

\section{Center of Gravity of the Human Brain}

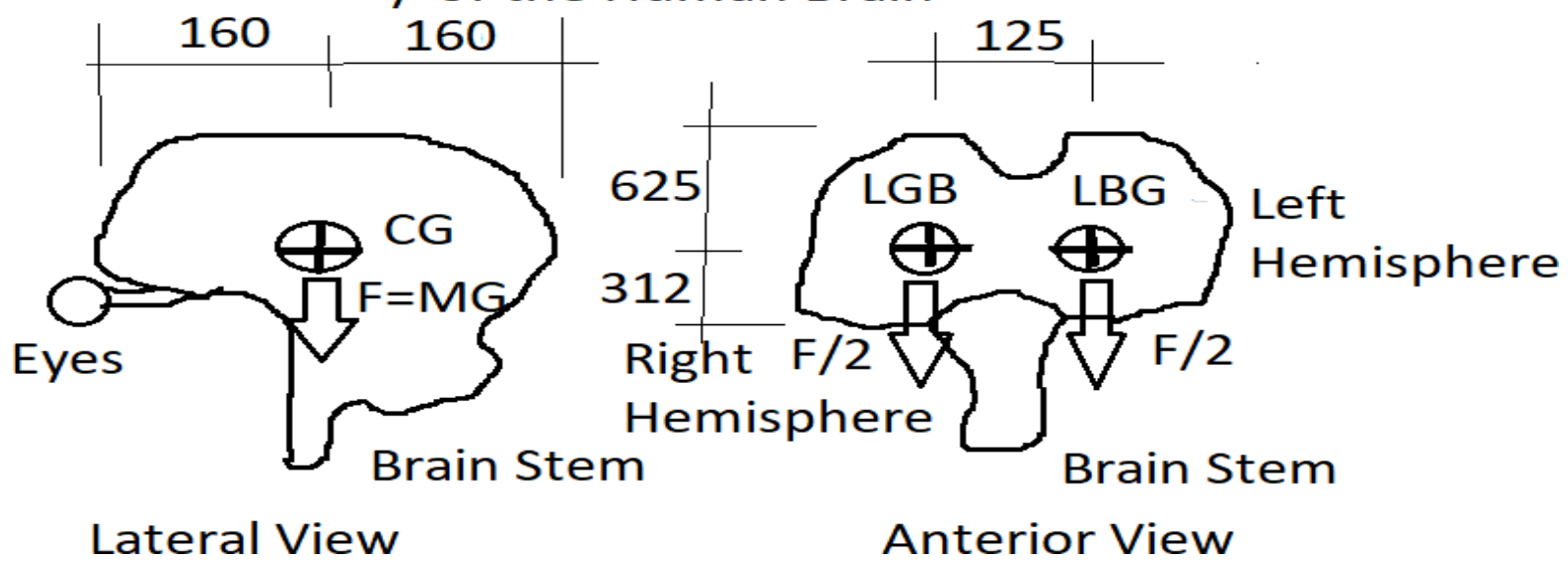




\section{$\mathrm{F}=\mathrm{MG}$}

$=1350(6.67)$

$=9.00$

$=\mathrm{c}^{2}$

$\mathrm{M}=4$

$\mathrm{F}=4(6.67)$

$=8 / 3=\mathrm{SF}$

$\mathrm{F}=\mathrm{MG}=\mathrm{c}^{2}$

$\mathrm{M} / \mathrm{c}^{2}=\mathrm{G}$

Clairnaut

$\mathrm{d}^{2} \mathrm{E} / \mathrm{dt}^{2}-\mathrm{G}=0$

$\mathrm{E}=\mathrm{G}^{3} / 3=6.67^{3} / 3=9.8913$

$\mathrm{t}=1010 \sim 10.10=$ Density of the human body.

$\mathrm{t}=\mathrm{KE}=1 / 2 \rho \mathrm{v}^{2}$

$=1 / 2 \rho(1 / \sqrt{ } 2)^{2}$

$=\rho / 4$

$4 \mathrm{t}=\rho=1.010$ Density of the human body

$\mathrm{t}=252=$ Period $\mathrm{T}=1 / \mathrm{t}$

$\mathrm{t}^{2}=252$

$\mathrm{t}=0.502=1 / 2=\mathrm{t}_{\min }$ of the Golden Mean Parabola $2 \mathrm{t}-1=0$

Mom=F x d

$=1585=\mathrm{c}^{2} \mathrm{x} \mathrm{d}$

$\mathrm{d}=\mathrm{s}=176$

$\mathrm{E}=(1-\operatorname{Ln} \mathrm{t})^{7}$

$-1.25^{1 / 7}=1-\operatorname{Ln~t}$

Ln $\mathrm{t}=1.122$

$\mathrm{t}=31.03 \sim 31=12$ th Prime Number

Gauss's Equation

$\operatorname{Lim} x \rightarrow \infty \pi(x) /[x / \operatorname{Ln} x\}=12 / 343=132.9 \sim 133=4 / 3=\mathrm{S}$

For the Brain:

$\mathrm{M}=1350$

$\mathrm{R}=0.85 \mathrm{ohms}$

$\mathrm{V}^{+}=105$

$\mathrm{i}=35$

$\rho=126$

$\mathrm{E}=112.2$

$\mathrm{PE}=\mathrm{Mc}^{2}$

$=1350 \mathrm{c}^{2}$

$=12133$

$\mathrm{t}=\mathrm{KE}=1 / 2 \mathrm{Mv}^{2}$
$=\mathrm{M} / 4=1350 / 4=337.5=\sim 1 / \mathrm{c}$

$\mathrm{SE}=\mathrm{t}^{2}-\mathrm{t}-1^{2}$

$337^{2}-337-1=112.6$

$\mathrm{E}=(1-\operatorname{Ln} \mathrm{t})^{7}$

112.56

$\mathrm{t}=31$ 12th Prime number

$\rho=\mathrm{M} / \mathrm{Vol}$.

$1350 / 126=1.0714$

$\operatorname{Ln}\left(\rho / \mathrm{Vol}^{2}\right)=1.0714 / 126$

$=0.85 \Omega$

$=\mathrm{R}$ for the Brain.

Vol $(625+312) \times(160+160)$

$=171840$

$\rho=\mathrm{M} / \mathrm{Vol}=1350 / 1718$

$=7.86$

$7.86 / 1.0714=7.33=1 / 1364=126 / 1718=\rho / \mathrm{Vol}$.

$7.33=\rho /$ Vol. $^{2}$

$=\rho / 171.8^{2}=23.11$

$\operatorname{Ln} 23.11=3.14=\pi$

$\mathrm{Vol} / \rho=171.840 / 126=136.4=1 / \rho \rho=0.733$

$\mathrm{V}=\mathrm{IR}$

$=4 / 3)(0.85)$

$=113.33$

$\mathrm{V}=\mathrm{iZ}$

113.33

$=\mathrm{i}(2)$

$=565$

$\mathrm{V}=\mathrm{E}=1 / \mathrm{t}$

$\mathrm{t}=176.5$

$E=(1-\operatorname{Ln} t)^{7}$

$=(1-\operatorname{Ln} 176.5)^{7}$

$=114.3$

$\mathrm{SE}=\mathrm{t}^{2}-\mathrm{t}-1^{2}$

$=176.5^{2}-175.6-1$

$=114.5$

$\mathrm{t}=\mathrm{KE}=1 / 2 \mathrm{Mv}^{2}$

$176.5=\mathrm{M} / 4$

$\mathrm{M}=706 \sim 707=1 / \sqrt{ } 2$

$\mathrm{PE}=\mathrm{Mc}^{2}$

$=(1 / \sqrt{ } 2) \mathrm{c}^{2}$

$=6.35=1 / 1575=1 /$ Moment

$\mathrm{TE}=\mathrm{PE}+\mathrm{KE}+\mathrm{SE}$

$=6.35+176.5+114 .=297 \sim \mathrm{c}$ 


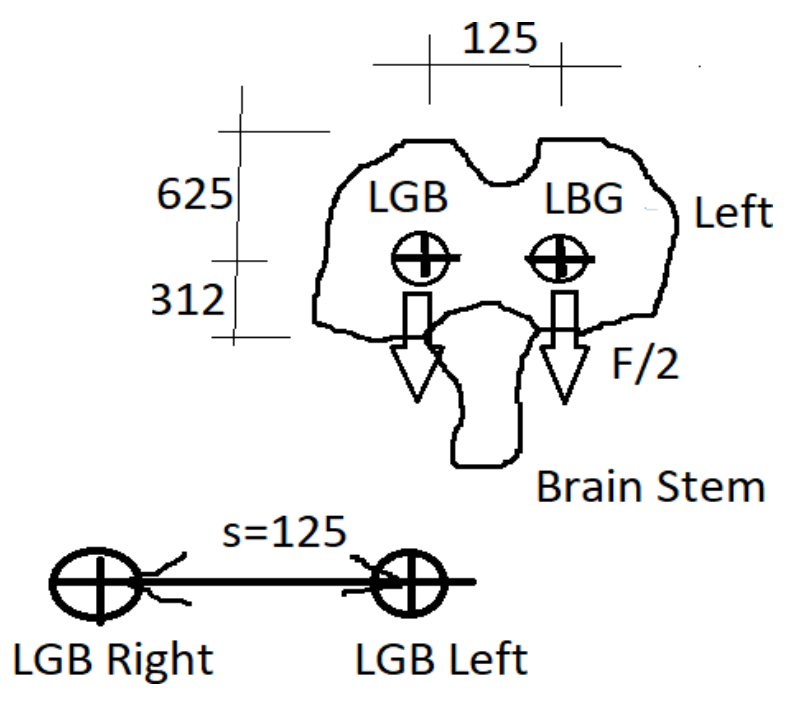

Figure 10: Evolving Human Brain. Centre of Gravity separates until critical point.

$\mathrm{ds} / \mathrm{dt}=\mathrm{v}=125 / 2=63.25=1 / 81=1 / \mathrm{c}^{4}=\mathrm{E} / \mathrm{c}^{2}=\mathrm{Mc}^{2}$

$\mathrm{E}=\mathrm{Mc}^{4}$

$=4(81)=324$

$1 / \mathrm{E}=\mathrm{t}=308.6 \sim 309$

Now,

$1 / 6.325=125=\mathrm{s}$

$1 / \mathrm{ds} / \mathrm{dt}=\mathrm{s}$

$1 / \mathrm{s}=\mathrm{ds} / \mathrm{dt}$

Integrate

$\int 1 / \mathrm{s}=\int \mathrm{ds} / \mathrm{dt}=\mathrm{s}$

$\operatorname{Ln} \mathrm{s}=\mathrm{s}$

$(\mathrm{s}-\mathrm{Ln} \mathrm{s})=(1-\operatorname{Ln} 1)$

$=1$

$=\mathrm{t}=\mathrm{E}$

$1 / \mathrm{s}=\operatorname{Ln~} \mathrm{s}$

Derivative

$-\mathrm{s}^{-2} /-2=1 / \mathrm{s}$

$1 /\left(2 \mathrm{~s}^{2}\right)=1 / \mathrm{s}$

$1 /(2 s)=1$

$\mathrm{s}=1 / 2$ $\mathrm{s}=\mathrm{t}_{\min }$

$1 / \mathrm{s}=\mathrm{Ln} \mathrm{s}+\mathbb{C}$

$1 /(1 / 2)=\operatorname{Ln}(1 / 2)+\mathbb{C}$

$2=-0.693+\mathbb{C}$

$\mathbb{C}=2.693$

$=\mathrm{c}^{3}$

Equation of the mind:

$\mathrm{L}=\operatorname{Ln}(\mathrm{t})+\mathrm{c}^{3}$

\section{Another equation of the mind is:}

$\mathrm{S}=\mathrm{M}-\mathrm{E}=1 / 9-1 / 8=1 / 72$

$\mathrm{L}=\operatorname{Ln}(1 / 2)+2.693$

$=-0.693 / 72+2.693=2.68 \sim 2.67=\Pi$ Senses

$2 / 72=0.0327=1 / 26.8$

Continuing,

$\mathrm{SE}=\mathrm{t}^{2}-\mathrm{t}-1=$

$(1 / 2.693)^{2}-(1 / 2.693)-1=0.123=1 / 81=1 / c^{4}$

$\mathrm{SE}=1 / \mathrm{c}^{4}=1 / 9 \times 1 / 9=\mathrm{E}^{2}$

$E=\sqrt{ } 1 / c^{4}=1 / c^{2}$ Input and output of the human mind.

$=\mathrm{M}$

$\mathrm{E}=\mathrm{Mc}^{2}=\left(1 / \mathrm{c}^{2}\right)\left(\mathrm{c}^{2}\right)=1$

$\mathrm{E}=\mathrm{t}=1$

$E=(1-\operatorname{Ln} t)^{7}$

$1=(1-\operatorname{Ln} 1)^{7}$

$1=1-0$

$1=1$

True!

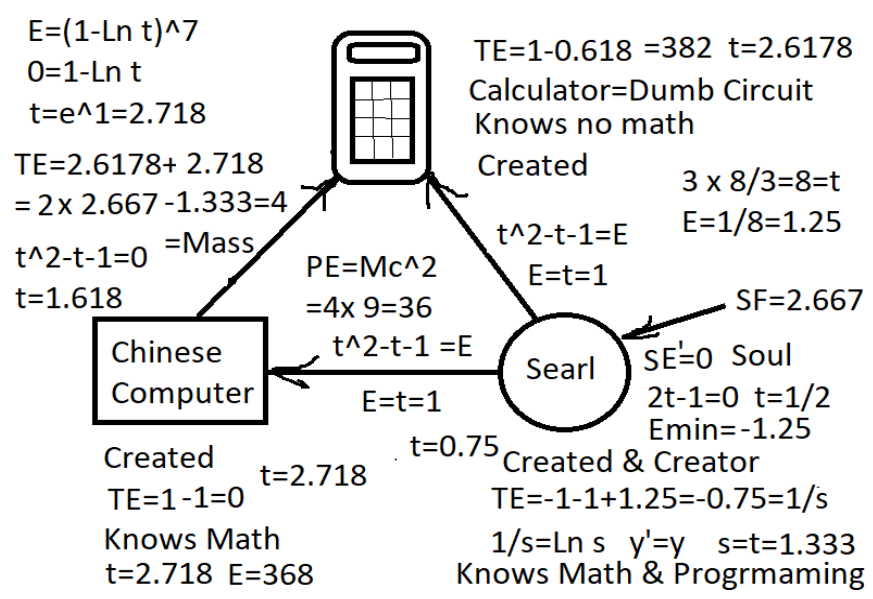




\section{Figure 11: The Chinese Room.}

$\mathrm{y}=\mathrm{y}^{\prime}$

$\mathrm{SE}=\mathrm{SE}$

$\mathrm{t}^{2}-\mathrm{t}-1=2 \mathrm{t}-1$

$t=3 E=5$

$\mathrm{E}=(1-\operatorname{Ln} \mathrm{t})^{7}$

$=(1-\operatorname{Ln} 3) 7$

$=9.07 \sim \mathrm{c}^{2}$

$t^{2}-t-1=c^{2}$

$\mathrm{t}=2.67 ; 3.67$

2.67 $=\mathrm{SF}=\Pi$ Senses

$(1 / 2.693)^{2}-(1 / 2.693)-1=1233444=1 / 81=1 / \mathrm{c}^{4}$

$=1 / 9 \times 1 / 9=\mathrm{M}^{2}$

$\mathrm{PE}=\mathrm{Mc}^{2}$

$=\mathrm{c}^{2} \times \mathrm{c}^{2}$ $=c^{4}$

$\mathrm{t}=1 / \mathrm{c}^{4}$

$\mathrm{M}=\mathrm{c}^{2}=\mathrm{Ln} \mathrm{t} \mathrm{t}=8 \mathrm{E}=1 / 8$

$\mathrm{t}^{2}-\mathrm{t}-1=(1 / 81)^{2}-(1 / 81)-1=-1.012$ Density of the human body

$\mathrm{E}=1.01 \sim 1$

$=\mathrm{t}=1$

\section{Conclusion}

We see that the mind, body, and soul evolved to match up with the universal signal.

\section{References}

1. Dennett, DC. Consciousness Explained. Back Bay Books NY 1991.

2. Cusack, PTE. Path of consciousness. (submitted)

3. Cusack, PTE. Astrotheology, Cusack's Universe. J of Phys Math., OMI USA., 2016.

4. Paul TEC. (2017), the Universal Parametric Equation. J Generalized Lie Theory Appl 11: 261.
This work is licensed under Creative Commons Attribution 4.0 License

To Submit Your Article Click Here: Submit Article

DOI: $10.31579 / 2690-8808 / 026$

\footnotetext{
Ready to submit your research? Choose Auctores and benefit from:

* fast, convenient online submission

* rigorous peer review by experienced research in your field

* rapid publication on acceptance

* authors retain copyrights

* unique DOI for all articles

* immediate, unrestricted online access

At Auctores, research is always in progress.

Learn more www.auctoresonline.org/journals/journal-of-clinical-casereports-and-studies
} 